\title{
Extreme events in the dispersions of two neighboring particles under the influence of fluid turbulence
}

\author{
R. Scatamacchia ${ }^{1,2}$, L. Biferale ${ }^{1}$, F. Toschi ${ }^{2,3}$ \\ ${ }^{1}$ Dept. Physics \& INFN, Univ. Tor Vergata, Via della Ricerca Scientifica 1, 00133 Rome, Italy. \\ ${ }^{2}$ Dept. Physics, Eindhoven Univ. of Technology, $5600 \mathrm{MB}$ Eindhoven, The Netherlands. \\ ${ }^{3}$ Dept. Mathematics and Computer Science Eindhoven Univ. of Technology, \\ 5600 MB Eindhoven The Netherlands \& CNR-IAC, Via dei Taurini 19, 00185 Rome, Italy.
}

(Dated: October 29, 2018)

\begin{abstract}
We present a numerical study of two-particle dispersion from point-sources in $3 D$ incompressible Homogeneous and Isotropic turbulence, at Reynolds number $R e \simeq 300$. Tracer particles are emitted in bunches from localized sources smaller than the Kolmogorov scale. We report the first quantitative evidence, supported by an unprecedented statistics, of the deviations of relative dispersion from Richardson's picture. Deviations are due to extreme events of pairs separating much faster than average, and of pairs remaining close for long times. The two classes of events are the fingerprint of complete different physics, the former being dominated by inertial subrange and large-scale fluctuations, while the latter by the dissipation subrange. A comparison of relative separation in surrogate white-in-time velocity field, with correct viscous-, inertial- and integral-scale properties allows us to assess the importance of temporal correlations along tracer trajectories.
\end{abstract}

The relative separation of pairs of fluid particles by turbulence has been first addressed by L.F. Richardson [1] in a pioneering paper in 1926 (see [2, [3] for recent reviews). The main question is simple and fundamental: given a pair of particles released, at time $t_{0}$, at a small separation $\boldsymbol{r}_{0}$ (smaller of the Kolmogorov dissipative scale, $\eta$ ) what is the probability to find the pair at any given distance, $r$, at a later time $t$ ? In the case of isotropic and homogeneous turbulence (HIT) the probability density function (PDF), $P\left(\boldsymbol{r}, t \mid \boldsymbol{r}_{0}, t_{0}\right)$, of pair separations depends on the amplitude of $\boldsymbol{r}(t)$ only. Moreover, asymptotically it should become independent of the initial condition, $P(r, t)$.

The knowledge of this distribution is of utmost importance for many applied studies in geophysics [4 [6], being connected also to risk-evaluations in environmental disasters. Moreover, it constitutes a highly non trivial statistical problem, due to its intrinsic non-stationary nature which connects Lagrangian properties, i.e. velocity fluctuations evaluated at the particle positions, along their whole past history 7]. Richardson proposed to model the particle separation, at inertial subrange distances $\eta \ll r \ll L_{0}$, as a diffusive process characterized by an effective turbulent diffusivity, $D_{\text {Ric }}(r)=\frac{1}{2} \frac{d\left\langle r^{2}\right\rangle}{d t}$, estimated empirically to follow a 4/3-law:

$$
D_{\text {Ric }}(r) \sim \beta r^{4 / 3} .
$$

Here $L_{0}$ is the large-scale of the flow and $\beta=k_{0} \epsilon^{1 / 3}$, where $k_{0}$ is a dimensionless constant and $\epsilon$ is the turbulent kinetic energy dissipation. It is easy Richardson's work with the Kolmogorov 1941 theory [8] by following the dimensional estimate [7, 9]:

$$
D_{\text {Ric }}(r) \sim \tau(r)\left\langle\left(\delta_{r} v\right)^{2}\right\rangle,
$$

where $\tau(r) \sim \epsilon^{-1 / 3} r^{2 / 3}$ is the eddy-turn-over-time at scale $r$ and $\left\langle\left(\delta_{r} v\right)^{2}\right\rangle=C_{0} \epsilon^{2 / 3} r^{2 / 3}$ is the second order
Eulerian longitudinal structure function. The resulting long time growth of the mean squared separation is:

$$
\left\langle r^{2}(t)\right\rangle=g \epsilon t^{3},
$$

$g$ being the Richardson constant uniquely determined in terms of $k_{0}$ [10 13]. Since 1], many experimental and numerical studies 12, 14 16] have focused on the subject, including also extensions to the case of particles with inertia [17 19]. There are not doubts that Richardson's picture captures some important features of turbulent dispersion, in particular concerning events with a typical separation of the order of the mean. On the other hand, fundamental questions exist about the capability of the theory to correctly predict also extremal events, i.e. those pairs with separation much larger/smaller than $\left\langle r^{2}(t)\right\rangle^{1 / 2}$. Such events are very difficult to measure, due to their low probability in absolute terms. Richardson's approach can be reinterpreted as the evolution of tracers in a stochastic Gaussian, homogeneous and isotropic velocity field, delta-correlated in time, with two-point correlation 20]: $\left\langle\delta_{r} v_{i}(t) \delta_{r} v_{j}\left(t^{\prime}\right)\right\rangle=\delta\left(t-t^{\prime}\right)\left[D_{\|}(r) \hat{r}_{i} \hat{r}_{j}+\right.$ $\left.D_{\perp}(r)\left(\delta_{i j}-\hat{r}_{i} \hat{r}_{j}\right)\right]$, where the longitudinal and transverse second order structure functions $D_{\|}(r)$ and $D_{\perp}(r)$ are such that $D_{\perp}(r)=D_{\|}(r)+1 / 2 r \partial_{r} D_{\|}(r)$, by incompressibility. Under this assumption, the evolution of $P(r, t)$ is closed and local: [7, 20]:

$$
\partial_{t} P(r, t)=r^{-2} \partial_{r} r^{2} D_{\|}(r) \partial_{r} P(r, t) .
$$

Whenever the eddy-diffusivity has a power-law behavior, $D_{\|}(r)=D_{0} r^{\xi}$ with $0 \leq \xi<2$, the above equation with initial condition $P\left(r, t_{0}\right) \propto \delta\left(r-r_{0}\right)$ can be solved in terms of special functions 21, 22]. So doing, it admits the celebrated asymptotic large time form (independent of $\left.r_{0}\right)$ :

$$
P_{\text {Ric }}(r, t) \propto \frac{r^{2}}{\left\langle r^{2}(t)\right\rangle^{\frac{3}{2}}} \exp \left\{-b\left(\frac{r}{\left\langle r^{2}(t)\right\rangle^{\frac{1}{2}}}\right)^{2-\xi}\right\} .
$$




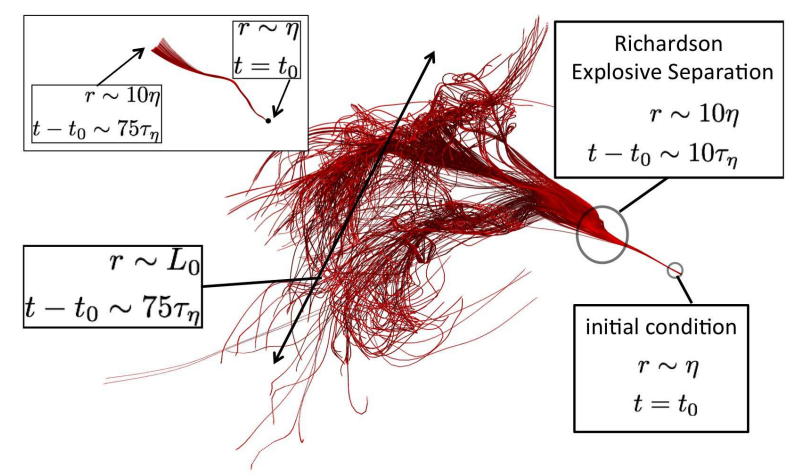

FIG. 1: Typical time history up to $t=75 \tau_{\eta}$ of a bunch emitted from a source of size $\sim \eta$. Inset: time history for the same duration of a bunch emitted in a different location and which does not separate. DNS are performed on a cubic fully periodic grid at $1024^{3}$ collocation points with a pseudospectral code, at a Reynolds-Taylor number, $R e_{\lambda} \sim 300$. For further details on the numerics see [12].

Here $b$ is a constant uniquely determined in terms of $D_{0}$ [21], which plays the same role of $\beta$ in eq. (11). In such $i d e-$ alized scaling scenario, tracer pairs separate in a explosive way forgetting their initial separation: $\left\langle r^{2}(t)\right\rangle \propto t^{2 /(2-\xi)}$, which reproduces Richardson's expression for $\xi=4 / 3$. When the turbulent flow is differentiable, i.e. $\xi=$ 2 , the PDF takes the log-normal form, $P\left(r, t \mid r_{0}, 0\right) \propto$ $\exp \left\{-\left(\log \left(r / r_{0}\right)-\lambda t\right)^{2} /(2 \Delta t)\right\}$, where $\lambda$ is the first Lyapunov exponent and $\Delta$ is connected to fluctuations of the strain matrix [7]. In the latter case, particles separate exponentially and the memory of the initial separation, $r_{0}$, remains at all times. The rate of separation is strongly fluctuating from point-to-point and from timeto-time being connected to the fluctuations of the Lyapunov exponents [7, 23].

The behavior of particle pairs in real flows can deviate from Richardson distribution because of different reasons. The four most important are: (i) temporal correlations of the underlying velocity fluid 9, 20, 24]; (ii) non-Gaussian velocity fluctuations; (iii) ultraviolet (UV) effects induced by the dissipation subrange, and (iv) infrared (IR) effects induced by the large-scale cut-off. These last two are of course connected to finite Reynolds effects [25].

The goal of this Letter is twofold. First, we want to understand and quantify the rate of occurrence of rare extreme events, i.e., of pairs that separate much more or much less than $\left\langle r^{2}(t)\right\rangle^{1 / 2}$. Second, we aim to assess the importance of temporal correlations for the pair statistics both in general and for extreme events, in particular.

We have performed a series of Direct Numerical Simulations (DNS) of HIT seeding the fluid with bunches of tracers, emitted in different locations, to reduce spatial correlations. Each bunch is emitted within a small region of size $\sim \eta$, in puffs of $2 \times 10^{3}$ tracer particles each. In a single run, there are 256 of such sources, emitting about 200 puffs with a frequency comparable with the inverse of the Kolmogorov time. We performed 10 different runs, following a total of $4 \times 10^{11}$ pairs, reaching an unprecedented statistics. In Fig. (1) we illustrate the complexity of the problem. We first notice the abrupt transition in the particle dispersion occurring at a time about $\sim 10 \tau_{\eta}$ after the emission when most of the pairs reaches a relative distance of the order of $\sim 10 \eta$, and the beginning of an explosive separation á la Richardson is observed. At at later time, there are many pairs with relative separations of the order of the box size $\sim 1000 \eta$, even though the mean separation is much smaller at those time lags. On the contrary, in the inset we show an example of a bunch with an anomalous history, due to tracers that travel close -at mutual distance of the order of $\eta$-, for very long times. This happens when pairs are injected in a space location where the underlying fluid has a small local stretching rate. Nevertheless, once the pairs reach inertial subrange separations, the bunch rapidly expands forgetting its initial delay and recovers at large times a spread distribution (not shown here).

To quantify this phenomenology, we show in Fig. (2) the right and left tails of $P(r, t)$ at different time lags, averaged over all emissions and over all point sources. The top panel shows the fastest separation events, with a clear exponential-like tail plus a sharp drop at a cut-off separation, $r_{c}(t)$, that evolves in time. The cut-off scale $r_{c}(t)$ is connected to pairs which are able to separate "very fast", and it is a clear indication of the change in the physics governing large excursions. It indicates the presence of a finite maximal propagation velocity: it is the signature of tracer pairs experiencing a persistently high relative velocity, which must be limited by the root-mean-squared velocity, $v_{r m s}$ [26]. To support this statement we show in the inset the evolution of $r_{c}(t)$ which is in good agreement with a linear behavior obtained using $v_{r m s}$ as traveling speed. Events beyond the cut-off scale are rare, and can be detected with high-statistics only.

The opposite limit of "very slow" events is also remarkable (see bottom panel of Fig. 2). Here we observe a bi-modal shape for $P(r, t)$ at almost all times: the left tail of pairs with mutual distance $r<\eta$ remains populated for time up to $\sim 60-70 \tau_{\eta}$, which is of the order of the large-scale eddy-turn-over-time, $T_{L}$. Pairs emitted in regions with a small stretching rate tend to stay together, reaching only very late the inertial subrange separations, and thus never experiencing a Richardsonlike dispersion. These pairs are governed by a log-normal distribution. This is a non-trivial result in tracers dispersion, that can not be brought back to small-scale clustering effects as those observed in the dynamics of inertial particles [27, 28]. 

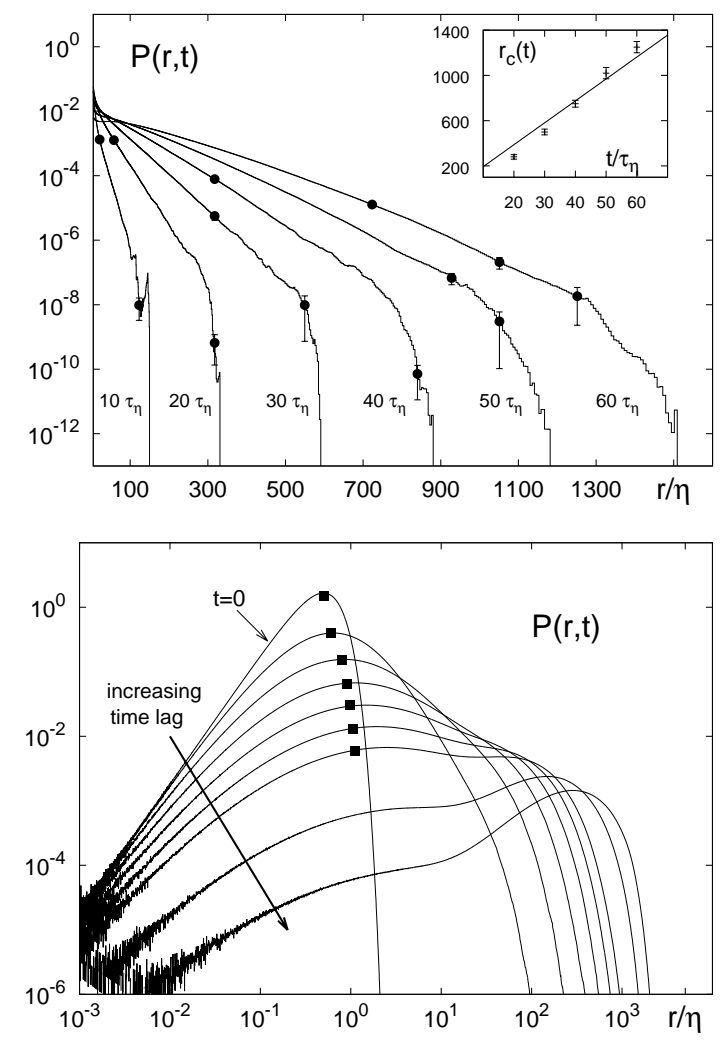

FIG. 2: Top: Log-lin plot of $P(r, t)$ at different times after the emission. For selected values of $r$, we show error bars, estimated from the statistical spread of different runs. Inset: evolution of the cut-off scale $r_{c}(t)$, the continuous line represents the ballistic motion $\propto v_{r m s} t$. Bottom: log-log plot of $P(r, t)$ for $t=(10,20,30,40,50,60,70,90,120) \tau_{\eta}$. Black squares indicates the peak observed for small separations.

In Fig. (3) we plot the same data of Fig. (2) but rescaled in terms of the variable $r_{n}(t)=r(t) /\left\langle r^{2}(t)\right\rangle^{1 / 2}$, and compared against the asymptotic prediction (5). Here, the deviations from Richardon's prediction becomes clear, showing evident discrepancies at large scales for all times. A more stringent test is obtained by showing these same PDFs but restricted to the scales in the inertial subrange, $30 \eta<r<300 \eta$ (inset). Clearly (5) is not satisfied. Previous studies could access events up to $r /\left\langle r^{2}(t)\right\rangle^{1 / 2}<3$ only (see [3]). Our study improves of four-five order of magnitudes (in probability) the intensities of detectable events, thus allowing to highlight strong deviations from Richardon's shape. Large discrepancies can be measured also on the left tails of $P(r, t)$, associated to very slow separating pairs (see also below).

Such departures from the ideal self-similar Richardson distribution needs to be better quantified, either in terms of finite Reynolds effects (break-up of self-similarity of the turbulent eddy diffusivity) or in terms of the neglected temporal correlations, or both.

To assess the importance of the former, we have inte-

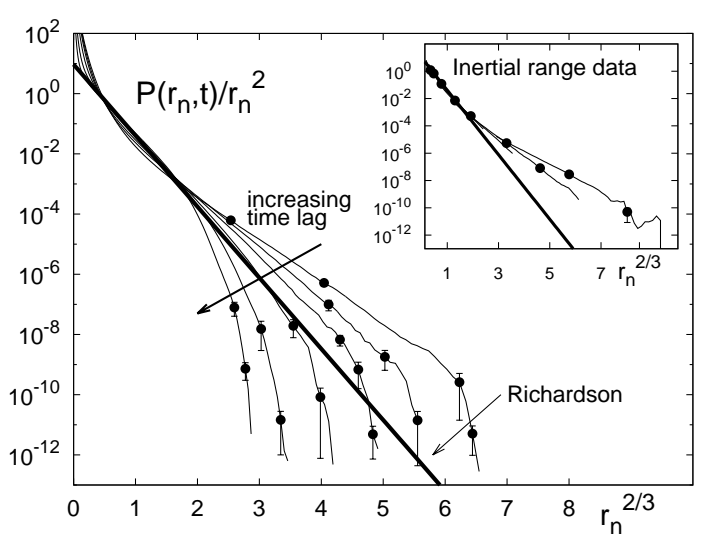

FIG. 3: Log-lin plot of $P\left(r_{n}, t\right)$ versus the rescaled variable $r_{n}$ (see text) for $t=(20,30,40,60,90,120) \tau_{\eta}$. The distribution $P\left(r_{n}, t\right)$ has been divided by a factor $r_{n}^{2}$ to highlight the large separation range. Richardson prediction (5) becomes timeindependent if rescaled in this way (solid curve). Inset: same PDFs plotted only for separations $r_{n}$ that, at any time lag, belong to the inertial subrange.

grated the Richardson equation (4) using an effective eddy-diffusivity $D^{e f f}(r)$ which improves (2) by including UV and IR cut-offs. Depending whether the separation distance falls in the viscous, inertial or integral range of scales, we must then have:

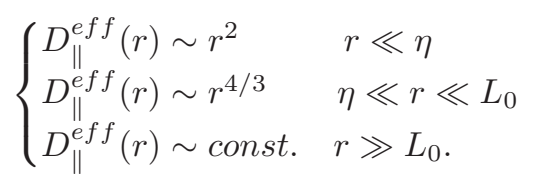

A widely used fitting formula that reproduces well the Eulerian data, and that matches the expected UV and IR scaling for both $\tau(r)$ and $\left\langle\left(\delta_{r} v\right)^{2}\right\rangle$, is obtained by following [29] :

$$
\left\langle\left(\delta_{r} v\right)^{2}\right\rangle=c_{0} \frac{r^{2}}{\left((r / \eta)^{2}+c_{1}\right)^{(2 / 3)}}\left[1+c_{2}\left(\frac{r}{L_{0}}\right)^{2}\right]^{-1 / 3}
$$

supplemented with a similar expression for the eddy turn over time: $\tau(r)=\frac{\tau_{\eta}}{\left((r / \eta)^{2}+c_{1}\right)^{-1 / 3}}\left[1+d_{2}\left(r / L_{0}\right)^{2}\right]^{-2 / 3}$. The dimensionless parameters $c_{0}, c_{1}, c_{2}$ are extracted from the Eulerian statistics, while the parameter $d_{2}$ is fixed such as to correctly reproduce the evolution of the mean square separation, $\left\langle r^{2}(t)\right\rangle$, over a time range

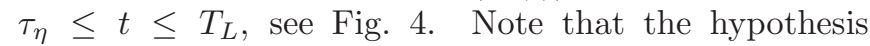
of Gaussian statistics still (implicitly) holds in this approach, since the velocity field distribution is fixed in terms of the second order moment only.

The solution, $P_{\text {eff }}(r, t)$, to the diffusive equation (4) using $D^{e f f}(r)$ is shown in Fig. 5 and compared with the DNS data. Despite the excellent agreement between $\left\langle r^{2}(t)\right\rangle$ obtained from DNS and the one obtained using the stochastic model (4) and (6), the far tails are still different. Self-similarity is broken by the introduction of 


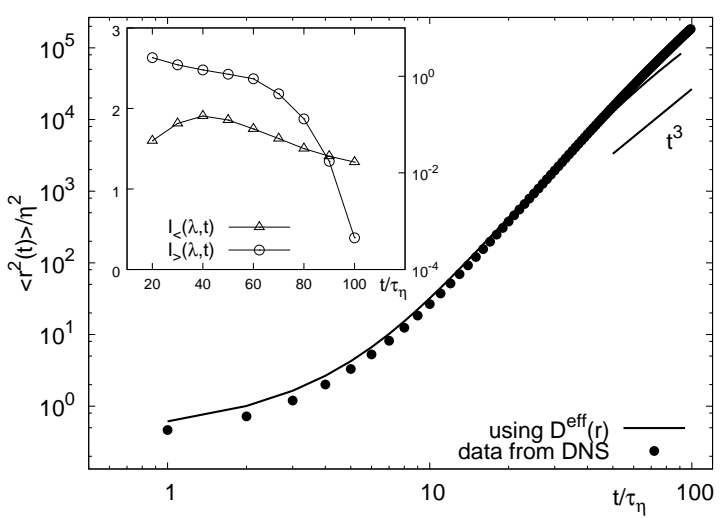

FIG. 4: Log-log plot of $\left\langle r^{2}(t)\right\rangle$ from DNS data, and from the diffusive evolution with eddy diffusivity (6). Inset: time evolution of the relative probability to observe a large excursion, $I_{>}(\lambda, t)$ (right $y$-scale), or small excursion $I_{<}(\lambda, t)$ (left $y$-scale) for $\lambda=3$.

UV and IR cutoffs in Eqn. (6), and therefore $P_{\text {eff }}(r, t)$ no longer rescales at different times: this is at variance with Richardson idealized picture, and clearly goes in the direction of real turbulent flows. For large separations the agreement with the DNS data is qualitatively better but still quantitatively off, particularly when focusing on the sharp change at the cut-off scale $r_{c}(t)$ which is still absent in the evolution given by (6). This is a key-point showing that to reproduce the observed drop in the PDF for large excursions, it is not enough to impose a saturation of eddy-diffusivity for large $r$. The behavior of pair dispersion must then be crucially dependent on the nature of temporal correlations of the Lagrangian turbulent velocity in the inertial subrange. Similarly, if we focus on the small separation tail, $P_{\text {eff }}(r, t)$ presents a slowly evolving peak for $r \leq \eta$ but quantitative agreement is again not satisfactory yet (inset of Fig. (5). We understand such departure as the effects of assuming a Gaussian velocity statistics underlying the evolution of $P_{\text {eff }}(r, t)$, which is blatantly wrong because of turbulent small-scale intermittency. To further quantify the departure of the modified Richardson description from the real data we measured the cumulative probability to have a couple at large separation $r^{*}(\lambda)=\lambda\left\langle r^{2}(t)\right\rangle^{1 / 2}$ normalized with the same quantity evaluated from Eqn. (5) using (6), namely $I_{>}(\lambda, t)=\int_{r^{*}(\lambda)}^{\infty} d r P(r, t) / \int_{r^{*}}^{\infty} d r P_{\text {eff }}(r, t)$; similarly, to evaluate the differences for small separation events, we use $r^{*}(\lambda)=1 / \lambda\left\langle r^{2}(t)\right\rangle^{1 / 2}$ and define: $I_{<}(\lambda, t)=\int_{0}^{r^{*}(\lambda)} d r P(r, t) / \int_{0}^{r^{*}} d r P_{\text {eff }}(r, t)$. The results

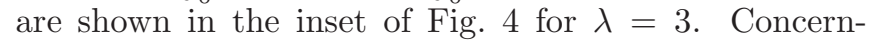
ing $I_{<}(\lambda, t)$, it shows that the evolution using $P_{\text {eff }}(r, t)$ underestimate of a factor 2 at small time-lags, $\sim 20 \tau_{\eta}$ the importance of the small-scale trapping properties, i.e. does not capture the strong intermittency of the regions where we have a small stretching rate. Only for very large

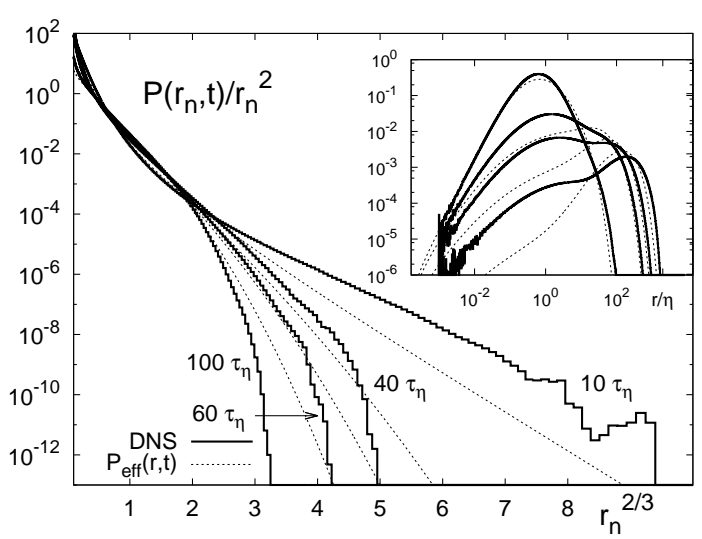

FIG. 5: Lin-log plot of $P_{\text {eff }}(r, t)$ as obtained from the integration of (5) with $D^{e f f}(r)$ (dashed) and the DNS data (solid line). Inset:log-log plot to highlight the slowest events (left tail).

times $\sim 100 \tau_{\eta}$ the left tail becomes comparable with the real ones. Concerning $I_{>}(\lambda, t)$ we measure first an underestimate of large separation events and later a strong overestimate, i.e. the delta-correlated approach behind the evolution of $P_{e f f}(r, t)$ does not capture the presence of the cut-off scale $r_{c}(t)$.

To directly quantify the importance of temporal correlations along tracer trajectories, we compare in Fig. (6) Lagrangian longitudinal velocity increments conditioned on particle separation distance $r$ :

$$
S_{\text {Lag }}(r, t)=\left\langle\left(\delta_{r(t)} v_{i} \cdot \hat{r}_{i}\right) \mid r(t)=r\right\rangle,
$$

with its Eulerian equivalent:

$$
S_{\text {Eul }}(r)=\left\langle\left|\delta_{r} v_{i} \cdot \hat{r}_{i}\right|\right\rangle .
$$

The figure shows that pairs that have separated fast have also a typical velocity difference higher than the Eulerian one, measured on the whole volume without conditioning on the history of particles. This is in agreement with what found before: intense separation events feel highly persistent velocity increments along their time history, and they cannot be trivially estimated starting from Eulerian statistics. Figure (6) also shows the opposite trend: pairs that have not separated much, at any given time, have Lagrangian velocity increments smaller than the Eulerian ones.

In conclusion, we have presented the first numerical study at very high statistics meant to explore rare events in turbulent dispersion. Both "fast" and "slow" separations show an important departure from the Richardsonlike inertial and idealized behavior of Eqn. (5). A step forward toward a better modelization is obtained by maintaining the assumption of time delta-correlation and Gaussian statistics but introducing an improved effective eddy-diffusivity kernel, which takes correctly into account both the viscous and integral scale physics. Doing so, a better qualitative agreement with real data is 


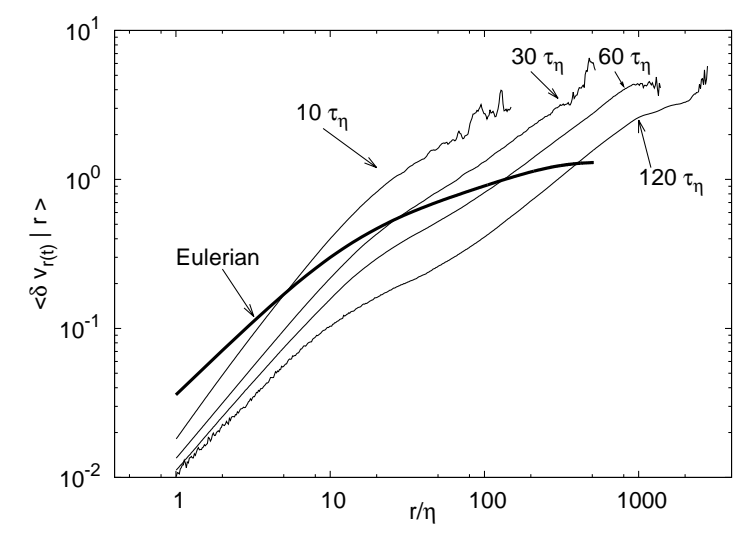

FIG. 6: Comparison between conditional Lagrangian (thin lines) and Eulerian (thick line) velocity increment moment.

observed. To further progress we will need to relax the Gaussianity assumption for the small-separation and the delta-correlated assumption for separations of the order of the integral scale. An attempt following the latter direction by using a Langevin-process for the evolution of the relative particles velocity, with different correlation times at different scales, has been recently proposed by [30]. Alternatively, a memory kernel can be introduced in order to overcome the presence of unphysical infinite speed events [24], characterizing pairs that move in a delta-correlated velocity field. For example, 1d model with telegraph equations shows similar maximum speed events [26].

We thank A.S. Lanotte for collaboration in a early stage of this work and M. Cencini, J. Bec and R. Benzi for discussions. We acknowledge the EU COST Action MP0806 "Particles in turbulence" and CINECA (Italy) within the HPC program ISCRA-Class A Project. This research was supported in part by the Project of Knowledge Innovation Program (PKIP) of Chinese Academy of Science, Grant No. KJCX2.YW.W10.

[1] L. F. Richardson, Proc. R. Soc. London A 110, 709 (1926).

[2] R. Benzi, A voyage through Turbulence, (Cambridge Univ. Press 2011, eds. P. Davison, Y. Kaneda, K. Moffatt and K. Sreenivasan).

[3] J. P. L. C. Salazar and L. R. Collins, Annu. Rev. Fluid Mech. 41, 405 (2009).

[4] P. J. Sullivan, J. Fluid Mech. 47, 601 (1971).

[5] M. Ollitrault, C. Gabillet, and A. Colin De Verdiére, J. Fluid Mech. 533, 381 (2005).

[6] J. H. LaCasce, J. Mar. Res. 68, 433 (2010).

[7] G. Falkovich, K. Gawȩdzki, M. Vergassola, Rev. Mod. Phys. 73, 913 (2001).

[8] U. Frisch, Turbulence, Cambridge University Press, Cambridge (1995).

[9] I. M. Sokolov, Phys. Rev. E 60, 5528 (1999).

[10] S. Ott and J. Mann, J. Fluid Mech. 422, 207 (2000).

[11] G. Boffetta, I.M. Sokolov, Phys. Rev. Lett. 88, 094501 (2002).

[12] L. Biferale, G. Boffetta, A. Celani, B.P. Devenish, A.S. Lanotte, and F.Toschi, Phys. Fluids 17, 115101 (2005).

[13] J. Schumacher, Phys. Rev. Lett. 100, 134502 (2008).

[14] M.C. Jullien, J. Paret, P. Tabeling, Phys. Rev. Lett. 82, 2872 (1999).

[15] F. Nicolleau and J.C. Vassilicos, Phys. Rev. Lett. 90, 024503 (2003).

[16] N. Ouellette, H. Xu, M. Bourgoin, E. Bodenschatz, New J. Phys. 8, 109 (2006).

[17] J. Bec, L. Biferale, A.S. Lanotte, A. Scagliarini, and F. Toschi, J. Fluid Mech. 645, 497 (2010).

[18] I. V. Derevich, Fluid Dyn. 43, 357 (2008).

[19] I. Fouxon, and P. Horvai, Phys. Rev. Lett. 100040601 (2008).

[20] R. H. Kraichnan, Phys. Fluids 11, 945 (1968); Phys. Fluids 9, 1937 (1966).

[21] E. Balkovski and V. Lebedev, Phys. Rev. E 58, 5776 (1998).

[22] A. Bennett, Lagrangian fluid dynamics Cambridge University Press, Cambridge (2006).

[23] J. Bec, L. Biferale, G. Boffetta, M. Cencini, S. Musacchio, and F. Toschi, Phys. Fluids 18, 091702 (2006).

[24] V. Ilyin, I. Procaccia and A. Zagorodny, Phys. Rev. E. 81,030105 (2010).

[25] B. L. Sawford, P.K. Yeung, and J. F. Hackl, Phys. Fluids 20, 065111 (2008).

[26] T. Ogasawara and S. Toh, J. Phys. Soc. Jpn. 75, 083401 (2006).

[27] J. Bec, L. Biferale, G. Boffetta, A. Celani, M. Cencini, A.S. Lanotte, S. Musacchio, and F. Toschi, J. Fluid Mech. 550, 349 (2006).

[28] L. Biferale, A. Scagliarini and F. Toschi, Phys Fluids 22, 065101 (2010).

[29] C. Meneveau, Phys. Rev. E 54, 3657 (1996).

[30] J. Bec, Talk presented at the Workshop New directions in Turbulence, KITPC, Beijing (2012). 